\title{
A chronometric analysis of strategy preparation in choice reactions
}

\author{
PETER DIXON \\ University of Alberta, Edmonton, Alberta, Canada \\ and \\ MARCEL ADAM JUST \\ Carnegie-Mellon University, Pittsburgh, Pennsylvania
}

\begin{abstract}
In this research, we investigated the process of preparing strategies for performing choice-reaction tasks. Before each choice-reaction trial, subjects were shown a cue that indicated features of the stimulus-response mapping to be used on the upcoming trial. Subjects used this cue to specify their strategy for responding to the stimulus. The time needed for specifying the strategy was measured by allowing subjects to control the cue presentation and surreptitiously recording how long they spent looking at the cue. The experiments demonstrated that the time to prepare a strategy was a function of the number and nature of the strategy features that had to be specified; simple uncertainty about the possible strategies had little direct effect. The results disconfirmed a serial model in which the time to prepare a strategy is the sum of the times to specify the individual strategy features. A mixed serial-parallel model was proposed as an alternative.
\end{abstract}

In the present article, we investigate the process by which people prepare strategies for performing simple speeded tasks. The term strategy is used to refer to the internal representation of the perceptual, cognitive, and motor operations needed to perform an information-processing task. The representation specifies the kind of perceptual analysis needed, the way in which the perceptual information is to be transformed, the decision rules to be used, and the responses to be generated (cf. Logan \& Zbrodoff, 1982; Logan, Zbrodoff, \& Fostey, 1983). The present notion of a strategy is similar to the concept of a motor program in that both specify the response to be made (see Keele \& Summers, 1976; Rosenbaum, 1980). However, a strategy contains information about the association of each of the responses with the various possible stimuli, in addition to information about how each response should be performed. In the experiments described here, we focused on how decision rules and responses are specified in strategies for choice reactions.

In this investigation, we used a strategy cuing paradigm, as developed by Dixon $(1979,1981)$. The paradigm is similar in some ways to the movement precuing technique de-

The authors wish to thank Patricia Carpenter, David Klahr, David Rosenbaum, Jeff Bisanz, Renée Elio, Gordon Logan, and Robert Proctor for comments on previous drafts. This research was supported by the United States National Institute of Mental Health (Grant No. MH29617 to the second author) and by the Natural Science and Engineering Research Council of Canada (Grant No. A8263 to the first author). Experiments 2 and 3 were reported previously at the 1978 Psychonomic Society Meeting in San Antonio, and in the first author's doctoral dissertation.

Requests for reprints should be sent to Peter Dixon, Department of Psychology, University of Alberta, Edmonton, Alberta, Canada, T6G 2E9. vised by Rosenbaum (1980), and to the strategy construction task used by Logan and his colleagues (Logan \& Zbrodoff, 1982; Logan et al., 1983). The technique also has a historical antecedent in the partial advance information paradigm (e.g., Davis, 1964; Leonard, 1958; Shaffer, 1966). In the strategy cuing paradigm, subjects were faced with a choice-reaction task in which the stimulus-response mapping varied from trial to trial. Prior to each choicereaction trial, subjects took as long as they wanted to examine a cue that specified the stimulus-response mapping for the forthcoming trial. The assumption underlying the paradigm is that subjects use this trial-initiation interval to specify a strategy appropriate for the given stimulusresponse mapping. Consequently, trial-initiation time can be used as an index of strategy-specification time.

Trial-initiation time was measured as a function of the number and nature of the alternative strategies possible within a block of trials. The rationale was that if all of the strategies shared some common features, then those features would not have to be specified on each trial; they could be specified earlier, at the beginning of the experiment or block of trials. An estimate of featurespecification time could be found by comparing initiation time across blocks of trials. For example, if all of the trials in a block required a right-hand response for the stimulus $\mathrm{X}$, and a left-hand response for an $\mathrm{O}$, then whatever strategy feature controlled the assignment of hand to stimulus could remain in force for the entire block, and would not have to be specified on each trial. On the other hand, if the assignment of hand to stimulus varied from trial to trial, information in the cue would have to be used to specify the strategy feature during the initiation time interval on each trial. Thus, the difference in initiation 
time between the two blocks would reflect the time to specify the hand-to-stimulus feature.

This paradigm is analogous to the movement precuing technique developed by Rosenbaum (1980). His subjects performed a choice-reaction task in which features of a response movement were cued $3 \mathrm{sec}$ prior to presentation of a stimulus. Reaction times were examined as a function of the number and nature of the movement features that remained to be specified on the basis of the stimulus. The cue in Rosenbaum's (1980) task allowed features to be specified in the motor program, in the same way that the blocking manipulation in the present task allowed features to be specified in a strategy. However, the mental representations being constructed in the two tasks were somewhat different. Rosenbaum's subjects prepared a motor program for a particular response, whereas in the present task, subjects prepared a strategy with two possible responses, not just one. Preparing a choicereaction strategy may also involve preparing motor programs for the responses, but it also involves specification of the mapping of stimuli to those responses.

The strategy cuing procedure is also similar to the paradigm used by Logan and Zbrodoff (1982; Logan et al., 1983). For instance, Logan and Zbrodoff (1982) presented a cue prior to a choice-reaction stimulus that could be used to form a more efficient strategy for responding to the stimulus. Reaction time was measured as a function of the delay between the cue and the stimulus, under the assumption that, with longer delays, subjects would have more of an opportunity to prepare and use the efficient, cue-based strategy. The principal difference between Logan's procedure and that used here is in the control of the interval between the cue and the trial. In Logan's procedure, the interval was varied by the experimenter, and the length of the interval needed to specify a strategy was inferred from the pattern of response times. In the present task, the interval was controlled by the subject, and it was assumed that the subject would terminate the interval only after the strategy had been constructed.

In this article, we address the following issues. First, in Experiments 1 and 2, we consider the extent to which initiation-time effects are related to the amount of strategy uncertainty within a block of trials. Initiation-time effects may be less interesting if they are simply a function of the number of possible strategies. In Experiment 3, we investigate whether initiation time is determined by cue encoding processes rather than strategy preparation. Trialinitiation time would be difficult to interpret if there were substantial effects of cue encoding variables. Finally, in Experiments 4 and 5, we consider in more detail the process by which strategy features are specified. In particular, we examine whether a serial-specification model is a plausible account of the process.

\section{EXPERIMENTS 1 AND 2}

In the first two experiments, we varied the number of possible strategies across blocks of trials to determine whether a systematic effect would be obtained on trial- initiation time. To demonstrate the generality of the results, Experiment 2 replicated Experiment 1, using a somewhat different task. A major goal of the two experiments was to evaluate the effect of uncertainty on trialinitiation time. A persistent issue in the motor programming literature is how to separate effects of movement specification from the more general effects of uncertainty about what the movement should be (e.g., Goodman \& Kelso, 1980; Megaw, 1972; Rosenbaum, 1980; Zelaznik, Shapiro, \& Carter, 1982). This literature suggests that it is important to demonstrate that strategy-preparation effects are independent of the amount of uncertainty about the strategy to be used. Otherwise, the results may simply represent a replication of the well-known effect of uncertainty in selection and choice. Such effects could be produced by a variety of mechanisms or factors (see, e.g., Smith, 1968), and there would be little reason to implicate the specification of strategy features as described here.

In Experiment 1, subjects made responses to the two stimuli by moving either their left or right index fingers from a home switch to one of four response switches placed at various distances (see Figure 1). There were eight possible stimulus-response mappings, given the constraints that the two stimuli were always assigned to different hands, and that homologous switches were used together. The conditions were designed to vary two strategy features: laterality, which governed the assignment of stimulus to left or right hand, and extent, which determined the extent of the response movement on each side. Conditions in Experiment 2 were constructed similarly, except that responses involved pressing one of eight buttons on which subjects rested their fingers (see Figure 2). Thus, the finger used to make the response was manipulated instead of response extent.

In both experiments, there were two, four, or eight possible stimulus-response mappings in a block of trials. The mappings were selected so that in the two-alternative condition, there was one strategy feature remaining to be specified during the trial-initiation interval, but in the fouralternative and eight-alternative conditions, there were two remaining features. Other features necessary to carry out the task would presumably be specified at the beginning of the block when subjects were informed of the nature of the trials. If it takes time to specify strategy features, there should be a difference in initiation time between the two-alternative condition and the four- and eightalternative conditions. The eight-alternative condition served as a control for any global effects of uncertainty; any effect of the number of strategies, independent of the number of strategy features, should show up as an increase from four to eight alternatives.

\section{Method}

Apparatus. In Experiment 1, subjects sat in front of an inclined response panel that contained 10 switches arranged in two columns (see Figure 1). The switches at the bottom of the two columns were home switches on which subjects rested their index fingers. Flat Sheldahl switches were used to minimize travel and effort. A speaker provided a soft click whenever a switch was pressed or released. Cues and stimuli were shown on a $30-\mathrm{cm}$ video monitor under the 


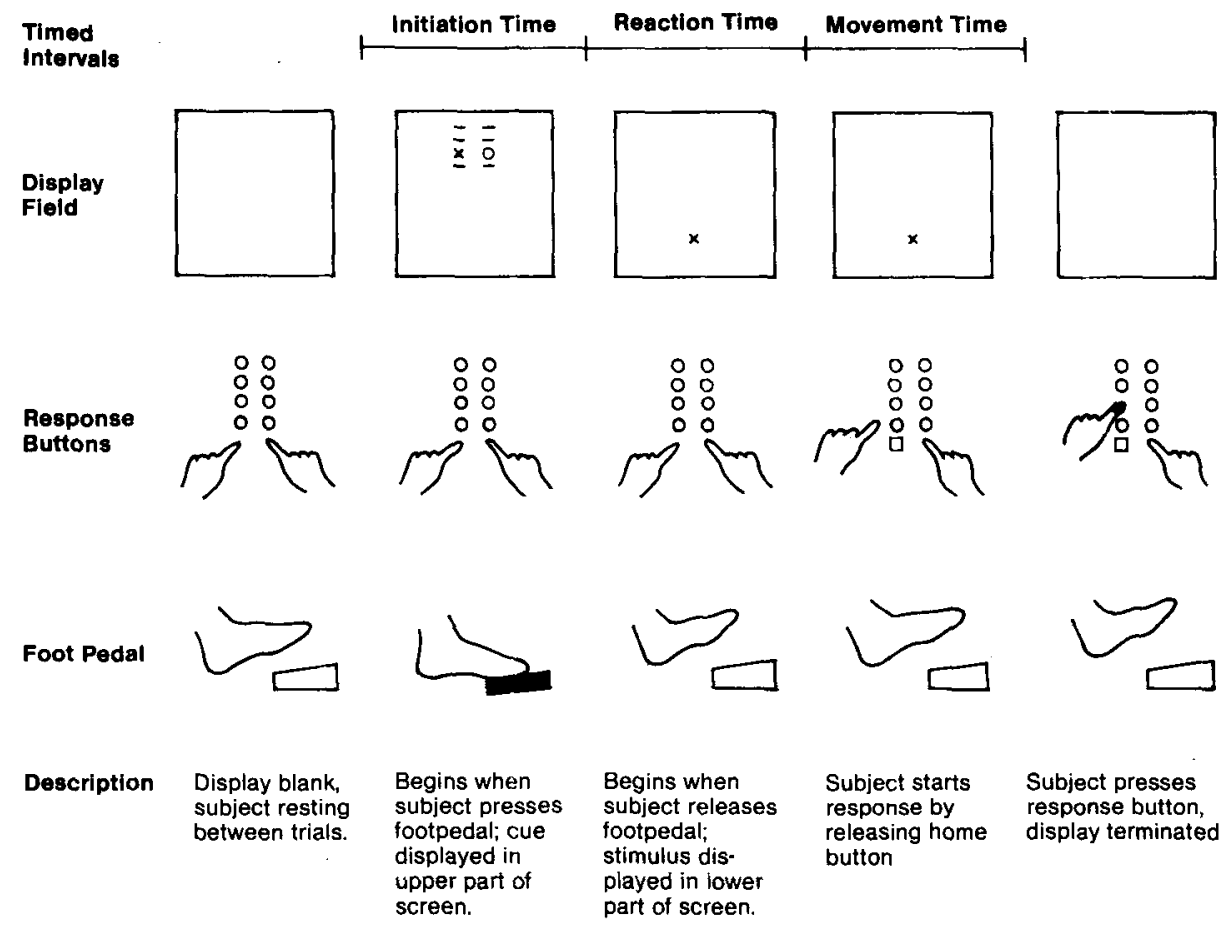

Figure 1. Sequence of events on each trial in Experiment 1.

control of an Apple II microcomputer system. In Experiment 2, subjects sat at an Iconix-type tachistoscope with their fingers resting on 8 pushbuttons. Cues and stimuli were presented in the upper and lower fields respectively.

Procedure. Figure 1 shows the sequence of events on a trial in Experiment 1. Between trials, subjects placed their index fingers on the pair of home switches. A fixation pattern, consisting of four dots at the corners of an imaginary rectangle, was shown on the screen to indicate the position of the cue and stimulus. Subjects then began each trial by pushing a foot pedal, which caused the cue to appear after $500 \mathrm{msec}$. The cue stayed on until the foot pedal was released, at which time the cue was replaced by the stimulus (either an $\mathrm{X}$ or an $\mathrm{O}$ ) just below the position of the cue. Responses were made by releasing a home switch and pressing one of the response switches in the column above it. Figure 2 shows the sequence of events on a trial in Experiment 2. The procedure was similar, ex-

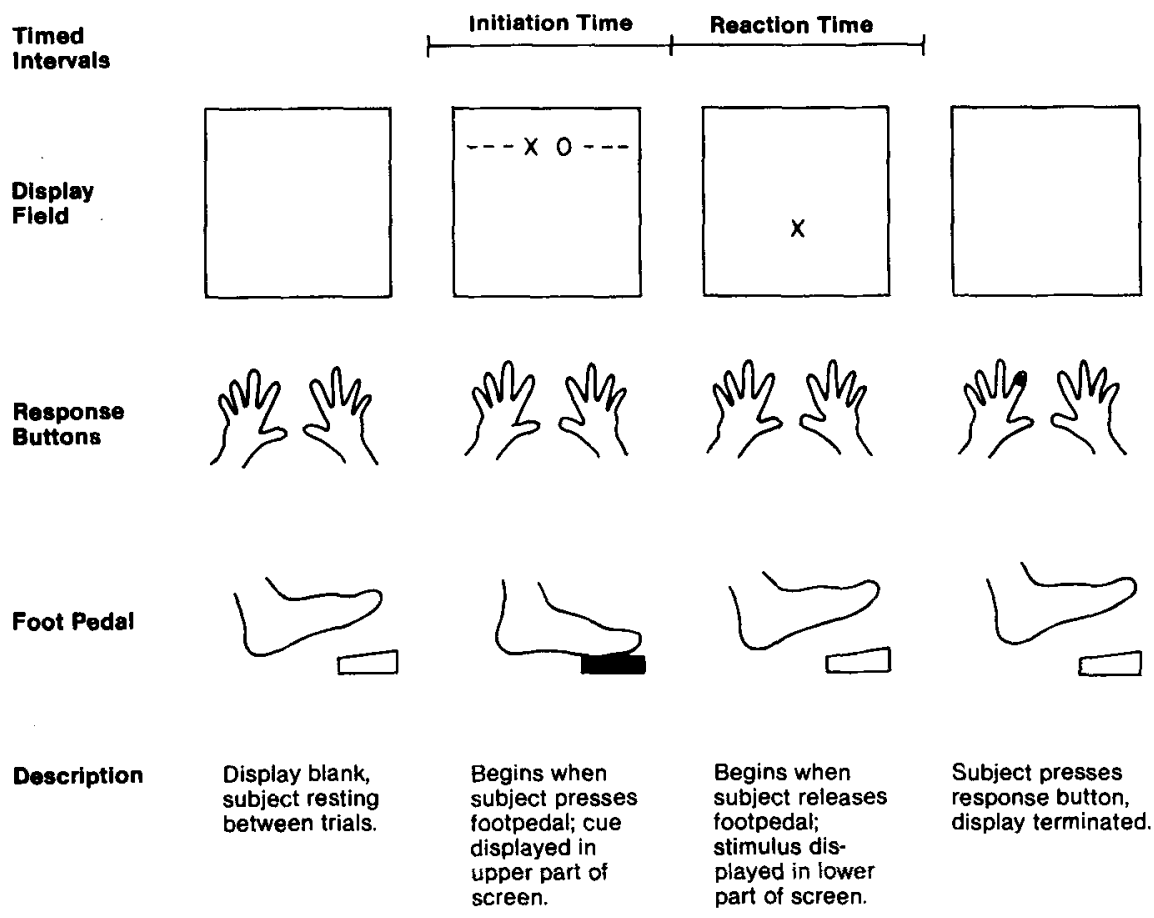

Figure 2. Sequence of events on each trial in Experiment 2. 
cept that responses were made by pressing a button with the appropriate finger.

In Experiment 1, initiation, reaction, and movement times were measured on each trial. Initiation time was the time that the subject spent looking at the cue after it had been presented on the screen. Reaction time was the time the subject took to begin making a response, and was measured from the onset of the stimulus to the release of a home switch. Movement time was the time between releasing a home switch and pressing a response switch. (Trials on which the wrong home switch was released were scored as errors.) In Experiment 2, only initiation and reaction times were measured; the reaction-time interval was terminated when the subject pressed one of the eight response buttons. In both experiments, subjects were instructed to respond as quickly as possible without making errors, but to take as long as they needed to study the cue.

In Experiment 1 , responses were numbered on the basis of the extent of the required movement. Type 1 responses involved pressing the nearest switch, Type 2 responses involved pressing the next nearest switch, and so on. In Experiment 2 the responses were numbered on the basis of which finger was used: Type 1 referred to responses with the index finger, Type 2 to responses with the middle finger, and so on. The terms Type 1, Type 2, and so on will be used to refer to the trials and cues associated with those responses.

Stimuli. In both experiments, the cues were schematic representations of the response panels constructed with the characters " $\mathrm{X}$," " $\mathrm{O}$," and "-." An " $\mathrm{X}$ " indicated which of the eight switches was the $\mathrm{X}$ switch and an " $\mathrm{O}$ " indicated which was the $\mathrm{O}$ switch; dashes ("-') stood for the unused switches. In Experiment 1, the symbols were arranged in two columns, representing the two columns of response switches. In Experiment 2, they were arranged in two adjacent rows. Cues were always symmetric in that the $\mathrm{X}$ and $\mathrm{O}$ occupied corresponding positions on either side. (See Figures 1 and 2 for examples.) In Experiment 1 , the characters subtended $0.4^{\circ}$ of visual angle at a typical viewing distance of $70 \mathrm{~cm}$; the centerto-center separation of the cue and the stimulus was $1.6^{\circ}$. In Experiment 2 , characters subtended $0.4^{\circ}$ of visual angle at a viewing distance of $57 \mathrm{~cm}$; the cue and stimulus were separated by $8.0^{\circ}$.

Conditions. Both experiments had three conditions. The twoalternative condition used only the two possible Type 1 responses. Half of the trials had the $X$ on the left and the $O$ on the right, and the $\mathrm{X}$ and $\mathrm{O}$ on the other half were reversed. Thus, the two stimulusresponse mappings differed only in the assignment of stimuli to the left- and right-hand responses. In the present terms, only a laterality feature remained to be specified in the strategy for each trial. The four-alternative condition used Type 1 and 2 responses. Thus, strategies varied not only in terms of a laterality feature, but also in terms of an extent feature (Experiment 1) or a finger feature (Experiment 2). The eight-alternative condition had eight stimulusresponse mappings representing all four types of responses. As in the four-alternative condition, two strategy features had to be specified on each trial. Each condition consisted of 48 trials, in which each possible cue in the condition was used equally often. For each cue, half the stimuli were Xs and half were Os. In Experiment 1, the 48 trials were run in a random order and preceded by 15 practice trials. In Experiment 2, the 48 trials were run in three randomly ordered blocks of 16; error trials were rerun later in the block. Subjects were informed of the nature of each condition before it began.

Subjects. In Experiment 1, subjects were 18 undergraduates at the University of Alberta. Three subjects were run with each of the six possible orders of the three conditions. In Experiment 2, subjects were 12 undergraduates at Carnegie-Mellon University and the University of Pittsburgh. Two subjects were run with each of the six possible condition orders. One subject in Experiment 2 was replaced because his initiation times averaged twice as long as anyone else's.
Analyses. The principal dependent measure was trial-initiation time. Parallel analyses were also performed on reaction time and movement time. In each case, the analysis was performed on the mean time for correct trials for each subject, condition, and response type. Outliers greater than three standard deviations above these means were excluded. In Experiment 1, these constituted $1.5 \%$ of the initiation times, $1.3 \%$ of the reaction times, and $1.5 \%$ of the movement times. In Experiment 2, these constituted $1.1 \%$ of the initiation times and $1.4 \%$ of the reaction times.

Because the number of trials was the same in each condition, the number of trials with each response varied across conditions. For instance, the two-alternative condition had 48 Type 1 trials, whereas the eight-alternative condition had only 12 . The effects of condition could well vary with response type, so analyses of variance were performed using planned contrasts to compare conditions within each response type. The main conclusions rest on the comparison of Type 1 trials, because these occurred in all three conditions.

\section{Results and Discussion}

Initiation times. Type 1 initiation times are shown in Figure 3 for Experiment 1 and in Figure 4 for Experiment 2 . In both experiments, there was a significant effect of condition that was due almost exclusively to the

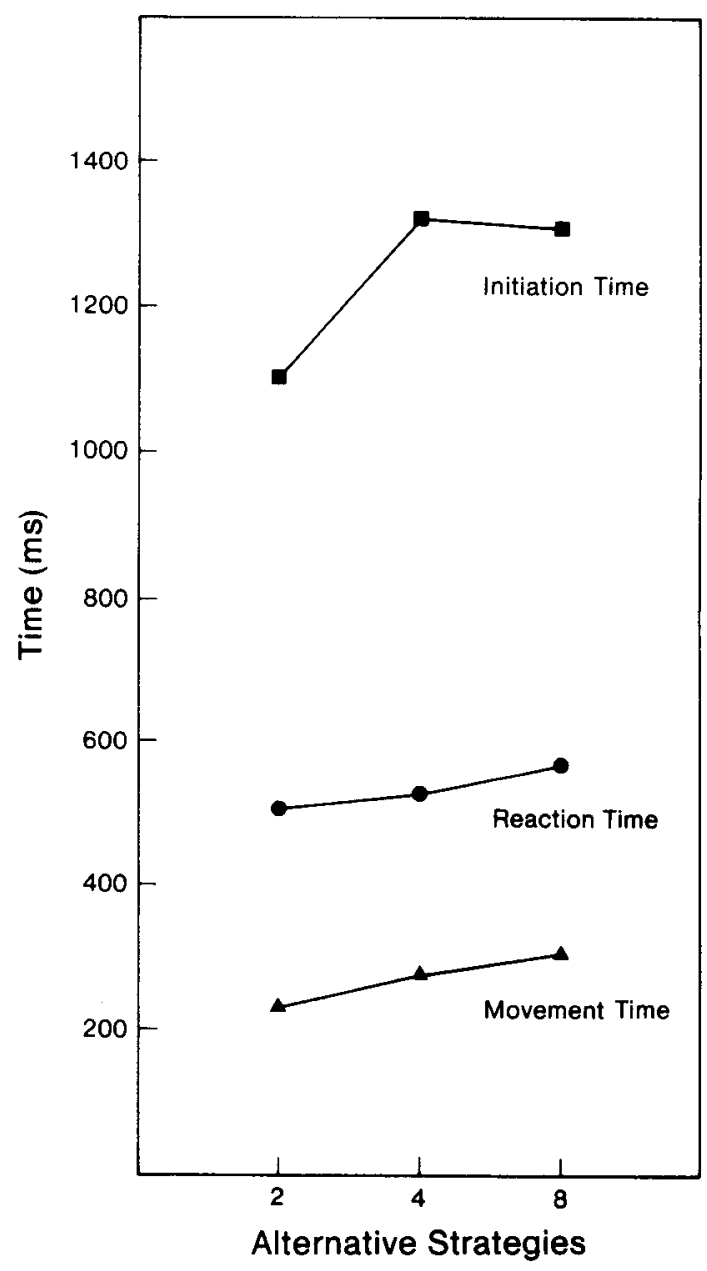

Figure 3. Mean initiation, reaction, and movement times for Type 1 trials in Experiment 1. 


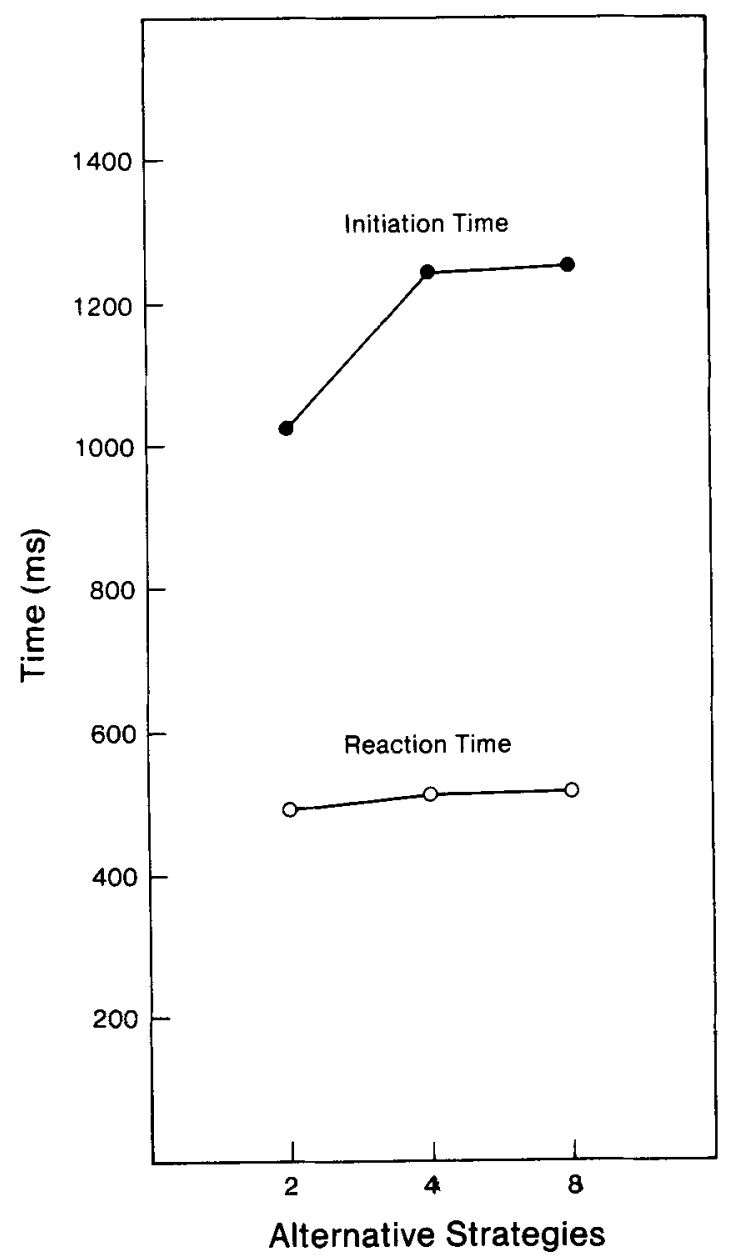

Figure 4. Mean initiation and reaction times for Type 1 trials in Experiment 2.

difference between the two-alternative condition and the four-alternative condition $[F(2,24)=5.33, p<.05$ in Experiment $1 ; F(2,12)=9.12, p<.005$ in Experiment 2]. There was a significant deviation from linearity in both cases $[F(1,12)=4.35, p<.06$ in Experiment 1 ; $F(1,6)=8.52, p<.05$ in Experiment 2], reflecting the fact that the four-alternative and eight-alternative conditions were almost equal.

These results support trial-initiation time as an index of strategy specification; initiation time was over 200 msec longer when two strategy features had to be specified than when only one had to be specified. It appers that more time is needed to specify the features of laterality and extent, or laterality and finger, than is needed to specify laterality alone. Moreover, there is little evidence in these results that uncertainty by itself affects initiation time: Even though there were twice as many strategies in the eight-alternative condition than in the four-alternative condition, there were no differences in initiation time. Presumably this reflects the fact that the two conditions required the same number of strategy features to be specified.
Initiation time comparisons also were made for the other response types. To begin with, the four-alternative condition and the eight-alternative condition were compared among Type 2 trials (see Tables 1 and 2). In Experiment 1 , there was no significant difference, but in Experiment 2 , the eight-alternative condition took longer $[F(1,6)=9.80, p<.05]$. The overall effect of response type was evaluated in the eight-alternative condition because this condition contained all possible responses. In both experiments, there was an effect of trial type, with initiation time being longer for Types 2 and 3 than for Types 1 and $4[F(3,36)=5.54, p<.005$ in Experiment $1 ; F(3,18)=4.72, p<.05$ in Experiment 2].

The trial type effects might be related to the relative discriminability of the cues. Type 1 and Type 4 cues may have been easy to distinguish in both experiments because the $\mathrm{X}$ and $\mathrm{O}$ occupied extreme positions in the cue array. In Type 2 and Type 3 cues, the $X$ and $O$ were in the interior of the array and may have been more difficult to discriminate. Consequently, more time may have been needed to decide which had been presented. Analogous

Table 1

Times (in Milliseconds) and Error Rates in Experiment 1

\begin{tabular}{lrrrr}
\hline & \multicolumn{4}{c}{ Trial Type } \\
\cline { 2 - 3 } Condition & 1 & 2 & 3 & 4 \\
\hline Two-Alternative & & & \\
Initiation Time & 1,104 & & \\
Reaction Time & 496 & & \\
Movement Time & 234 & & & \\
\% Errors & 7.5 & & & \\
Four-Alternative & & & & \\
Initiation Time & 1,324 & 1,438 & & \\
Reaction Time & 532 & 574 & & \\
Movement Time & 275 & 375 & & \\
\% Errors & 5.6 & 5.8 & & \\
Eight-Alternative & & & & \\
Initiation Time & 1,305 & 1,514 & 1,390 & 1,247 \\
Reaction Time & 572 & 619 & 567 & 537 \\
Movement Time & 299 & 398 & 406 & 425 \\
\% Errors & 5.1 & 9.3 & 7.4 & 8.8 \\
\hline
\end{tabular}

Table 2

Times (in Milliseconds) and Error Rates in Experiment 2

\begin{tabular}{|c|c|c|c|c|}
\hline \multirow[b]{2}{*}{ Condition } & \multicolumn{4}{|c|}{ Trial Type } \\
\hline & 1 & 2 & 3 & 4 \\
\hline \multicolumn{5}{|l|}{ Two-Alternative } \\
\hline $\begin{array}{l}\text { Initiation Time } \\
\text { Reaction Time } \\
\text { \% Error }\end{array}$ & $\begin{array}{r}1,028 \\
494 \\
2.6\end{array}$ & & & \\
\hline \multicolumn{5}{|l|}{ Four-Alternative } \\
\hline $\begin{array}{l}\text { Initiation Time } \\
\text { Reaction Time } \\
\% \text { Error }\end{array}$ & $\begin{array}{r}1,238 \\
513 \\
5.2\end{array}$ & $\begin{array}{r}1,336 \\
542 \\
3.8\end{array}$ & & \\
\hline \multicolumn{5}{|l|}{ Eight-Alternative } \\
\hline $\begin{array}{l}\text { Initiation Time } \\
\text { Reaction Time } \\
\% \text { Error }\end{array}$ & $\begin{array}{r}1,255 \\
510 \\
2.1 \\
\end{array}$ & $\begin{array}{r}1,460 \\
524 \\
6.9 \\
\end{array}$ & $\begin{array}{r}1,378 \\
523 \\
6.9 \\
\end{array}$ & $\begin{array}{r}1,255 \\
546 \\
6.2 \\
\end{array}$ \\
\hline
\end{tabular}


effects have been found, for example, in visual masking (e.g., Merikle \& Coltheart, 1972). This effect may also underlie the difference between the four-alternative and eight-alternative conditions for Type 2 trials in Experiment 2 . In the eight-alternative condition, Type 2 cues could potentially have been confused with Type 3 cues, and additional time may have been needed to discriminate the two. But in the four-alternative condition, Type 3 cues were not used, and there was no danger of confusing Type 2 and Type 3 cues. Here, subjects may have needed less time to decide that a Type 2 cue had been shown, and the total initiation times may have been shorter as a result.

Reaction and movement times. Identical analyses were also carried out on reaction time and movement time. Times for Type 1 trials are shown in Figures 3 and 4. In Experiment 1, both reaction time and movement time increased across conditions [for reaction time, $F(2,24)=$ $4.38, p<.05$; for movement time, $F(2,24)=11.52$, $p<.001]$. However, the same trend for reaction times in Experiment 2 was unreliable $[F(2,12)=2.72]$. A possible explanation for the effects in Experiment 1 is that, on some percentage of the trials, subjects initiated the trial without fully processing the cue. During the reaction and movement intervals, subjects would then have had to decide not only which stimulus was shown, but also which of several possible responses went with that stimulus. Depending on the condition, there would be two, four, or eight possible responses for each stimulus. The obtained results would then reflect an increase in reaction and movement times with response uncertainty on those few trials. The fact that no such effects were obtained in Experiment 2 suggests that these subjects were more careful about completely preparing their strategy before initiating the trial.

Analyses were also performed on reaction and movement times for different response types. For Type 2 trials, the four-alternative condition could be compared to the eight-alternative condition. This comparison was significant only for movement time in Experiment $1[F(1,12)$ $=7.18, p<.05]$. All four response types could be compared in the eight-alternative condition. There was an effect for both reaction and movement time only in Experiment 1 [for reaction time, $F(3,36)=9.98, p<.001$; for movement time, $F(3,36)=33.84, p<.0011$. Some of these effects may represent the particular demands of making the individual responses. For instance, longer movements took longer to make.

Errors. Error rates are shown in Tables 1 and 2. The overall error rate was $6.9 \%$ in Experiment 1 and $4.0 \%$ in Experiment 2, but there was no difference in error rate across conditions.

\section{Summary}

The main result of Experiments 1 and 2 was that trialinitiation time increased from two alternatives to four, but not from four alternatives to eight. This result validates trial-initiation time as a measure of strategy specification and simultaneously suggests that it is not influenced by the amount of uncertainty about strategies per se. There were two important qualifications on this conclusion. First, in Experiment 1, the number of possible strategies affected reaction and movement times. This suggests that subjects sometimes failed to specify completely their strategy during the initiation interval, and that there was some spillover of processing into the reaction and movement intervals. Second, in Experiment 2 there was a difference between the four-alternative condition and the eight-alternative condition for Type 2 trials. This might be related to cue discriminability, or it might mean that there was a residual effect of uncertainty that was not picked up in the Type 1 trial analysis.

\section{EXPERIMENT 3}

In Experiment 3, we tested the assumption that the observed initiation-time effects were due to the process of interpreting the cue and preparing a strategy, rather than to perceptual and encoding operations. We conceived of the initiation-time processing as consisting of two phases. First, the cue is perceived and represented internally. Presumably, these operations are relatively low level and automatic, and are largely unaffected by the meaning or interpretation of the cue (see Shiffrin \& Schneider, 1977). Second, that internal representation is interpreted and used to prepare a strategy. If this "interpretive" processing is the locus of the initiation-time effects in Experiments 1 and 2 , the results indicate that more interpretive processing is required when there are two unspecified features instead of one. However, another possibility is that some portion of the initiation-time effects were due to perceptual processing and encoding. Specifically, the time to encode the cue may increase with uncertainty about what cue might be presented (Biederman \& Zachary, 1970). A similar criticism has been made of the movement precuing technique (Zelaznik, 1978).

This issue was addressed in Experiment 3 by varying uncertainty about cues independently of uncertainty about alternative strategies. The two-alternative and fouralternative conditions from Experiment 2 were replicated in two ways. One way was similar to Experiment 2 in that only a single cue was used for each stimulus-response mapping. The other way was to use two different cues for each mapping. Thus, the same strategy features would have to be specified, but there would be additional uncertainty about what physical cue would be presented. If cue uncertainty affects initiation time, one should find an increase in initiation time under these circumstances, even though there is no change in the number and nature of the strategies.

There were four conditions in this experiment. The twoalternative/two-cue condition and the four-alternative/fourcue condition were replications of the two-alternative and four-alternative conditions from Experiment 2. The twoalternative/four-cue and four-alternative/eight-cue conditions were similar, except that two different cues were used 
to signal each stimulus-response mapping. Any difference between these new conditions and the old conditions could be attributed solely to the number of cues, not to the process of strategy specification. However, any difference between the two-alternative/four-cue condition and the four-alternative/four-cue condition could be attributed to the process of strategy specification, independent of uncertainty about the physical cue.

\section{Method}

Apparatus and Procedure. The procedure was generally the same as in Experiment 2. The apparatus was also similar, except that subjects had only four possible buttons to press, two on the left and two on the right.

Stimuli. The cues and stimuli were physically the same as in Experiment 2 . The major change from Experiment 2 was in the relationship between cues and strategies. In Experiment 2, each response button was associated with a single position in the cue array. But in Experiment 3, each response button was associated with two positions in the array. Type 1 and Type 2 cues both indicated index finger responses, and Type 3 and Type 4 cues both indicated middle finger responses. (Types $1,2,3$, and 4 refer to the same cues as in Experiment 2, even though they are not associated with distinct responses.)

Conditions. Each subject participated in the four conditions shown in Table 3. Two of the conditions (the two-alternative/two-cue and four-alternative/four-cue conditions) were identical to those in Experiment 2, except that Type 4 cues were used instead of Type 2 cues to indicate middle finger responses. In the two other conditions, each stimulus-response mapping was indicated by two different cues (the two-alternative/four-cue condition and the fouralternative/eight-cue condition). Cue arrays were always symmetric (i.e., the $\mathrm{X}$ and $\mathrm{O}$ were in corresponding positions on the two sides), as before. Each condition consisted of three blocks of 16 trials in which all cues and stimuli occurred equally often.

Subjects. Twelve undergraduates at Carnegie-Mellon University served as subjects. They were randomly divided into four groups, each of which had a different order of conditions. Across groups of subjects, each condition occurred once in each serial position in the experimental session.
Analysis. As in Experiments 1 and 2, the results were analyzed with planned comparisons within each cue type, using the mean initiation and reaction times for each subject and condition. Outliers greater than three standard deviations above these means were excluded $(2.1 \%$ of the initiation times and $0.8 \%$ of the reaction times).

\section{Results}

Initiation Times. As shown in Figure 5, trial-initiation time for Type 1 trials increased with the number of alternative strategies $[F(1,8)=91.63, p<.001]$. The effect of number of strategies independent of the number of cues was assessed by comparing the twoalternative/four-cue condition with the fouralternative/four-cue condition; there was a substantial difference $[F(1,8)=25.95, p<.001]$. There was also a small but significant effect of the number of cues per strategy $[F(1,8)=8.28, p<.05]$. The interaction between the number of cues and the number of strategies did not reach significance $[F(1,8)=3.32, p>.05]$. In sum, the number of strategies accounted for $92 \%$ of the total variance due to conditions, whereas the remaining two effects accounted for only about $8 \%$.

Other cue types showed similar effects (see Table 4). For Type 2 cues, the four-alternative/eight-cue condition took longer than the two-alternative/four-cue condition $[F(1,8)=20.04, p<.005]$, and in Position 4, the fouralternative/eight-cue condition was not significantly longer than the four-alternative/four-cue condition $[F(1,8)=$ $2.34, p>.05]$. The effect of different cue positions was evaluated in the four-alternative/eight-cue condition, since it included all possible cues. There was an overall effect of cue type $[F(3,24)=3.51, p<.05]$. The pattern of initiation times across cue types was roughly similar to that in Experiments 1 and 2, and may be related to the relative discriminability of the cues.

Table 3

Conditions Used in Experiment 3

\begin{tabular}{|c|c|c|}
\hline Condition & Possible Cues & Stimulus-Response Mapping \\
\hline Two-Alternative/Two-Cue & $\begin{array}{l}---X \text { O--- } \\
---O \text { X--- }\end{array}$ & $\begin{array}{l}X \rightarrow \text { left index }, O \rightarrow \text { right index } \\
\mathrm{O} \rightarrow \text { left index, } \mathrm{X} \rightarrow \text { right index }\end{array}$ \\
\hline Four-Alternative/Four-Cue & 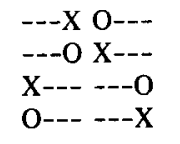 & $\begin{array}{l}\mathrm{X}-\text { left index }, \mathrm{O} \rightarrow \text { right index } \\
\mathrm{O} \rightarrow \text { left index } \mathrm{X} \rightarrow \text { right index } \\
\mathrm{X} \rightarrow \text { left middle, } \mathrm{O} \rightarrow \text { right middle } \\
\mathrm{O} \rightarrow \text { left middle, } \mathrm{X} \rightarrow \text { right middle }\end{array}$ \\
\hline \multirow[t]{2}{*}{ Two-Alternative/Four-Cue } & $\begin{array}{l}---X \quad O--- \\
--X--O--\end{array}$ & $\mathrm{X} \rightarrow$ left index, $\mathrm{O} \rightarrow$ right index \\
\hline & $\begin{array}{l}---O X X-- \\
--O--X--\end{array}$ & $\mathrm{O} \rightarrow$ left index, $\mathrm{X} \rightarrow$ right index \\
\hline \multirow[t]{4}{*}{ Four-Alternative/Eight-Cue } & $\begin{array}{l}---X \quad O--- \\
--X--O--\end{array}$ & $\mathrm{X}-$ left index, $\mathrm{O} \rightarrow$ right index \\
\hline & $\begin{array}{l}---O X X--- \\
--O--X--\end{array}$ & $\mathrm{O} \rightarrow$ left index, $\mathrm{X} \rightarrow$ right index \\
\hline & $\begin{array}{l}\mathrm{X}------O \\
-\mathrm{X}----\mathrm{O}-\end{array}$ & $\mathrm{X} \rightarrow$ left middle, $\mathrm{O} \rightarrow$ right middle \\
\hline & $\begin{array}{l}\mathrm{O}---\mathrm{-}-\mathrm{X} \\
-\mathrm{O}----\mathrm{X}- \\
\end{array}$ & $\mathrm{O}-$ left middle, $\mathrm{X} \rightarrow$ right middle \\
\hline
\end{tabular}




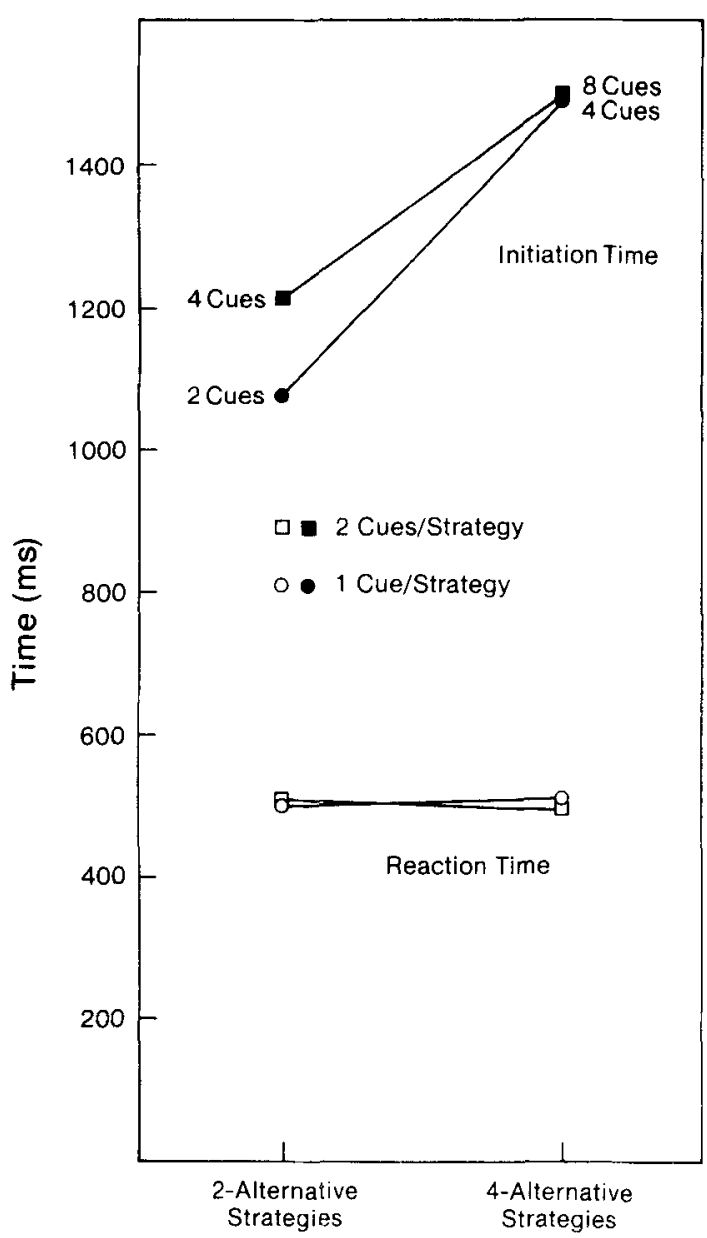

Figure 5. Mean initiation and reaction times for Type 1 cues in Experiment 3.
Reaction time and errors. Similar analyses were conducted for reaction time. There were no effects of condition, but there was a significant effect of cue type $[F(3,24)$ $=6.19, p<.005]$. The overall error rate was $2.7 \%$, and did not differ across conditions (see Table 4).

\section{Discussion}

These results indicate that simple uncertainty about which of several possible cues would be presented has only small effects on trial-initiation time. Presumably, uncertainty about the cues affects the encoding operations that construct the initial mental representation of the cue. The minimal effect of cue uncertainty suggests that encoding operations play only a small role in the observed initiation time effects. These results provide some assurance that the duration of encoding and representational processes are relatively invariant across conditions in this task.

This conclusion is consistent with a number of other sources of evidence. First, Logan and Zbrodoff (1982) varied the amount of processing needed to detect and discriminate a strategy cue, and found little effect on the time course of strategy preparation. Processes involved in interpreting the cue were thought to have much larger effects on the time to specify a strategy. Second, in Experiments 1 and 2, initiation time did not increase from four alternatives to eight, even though uncertainty about the physical cue increased. If the difference between the twoalternative and the four-alternative condition was due to cue uncertainty, one would have expected a similar increase from the four-alternative to the eight-alternative condition. Finally, in experiments that have manipulated stimulus uncertainty independently of response uncer-

Table 4

Times (in Milliseconds) and Error Rates in Experiment 3

\begin{tabular}{|c|c|c|c|c|}
\hline \multirow[b]{3}{*}{ Condition } & \multicolumn{4}{|c|}{ Cue Type } \\
\hline & 1 & 2 & 3 & 4 \\
\hline & (Index Finger & Responses) & (Middle Finger & Responses) \\
\hline \multicolumn{5}{|c|}{ Two-Alternative/Two-Cue } \\
\hline $\begin{array}{l}\text { Initiation Time } \\
\text { Reaction Time } \\
\% \text { Error }\end{array}$ & $\begin{array}{c}1,077 \\
498 \\
3.3\end{array}$ & & & \\
\hline \multicolumn{5}{|c|}{ Two-Alternative/Four-Cue } \\
\hline $\begin{array}{l}\text { Initiation Time } \\
\text { Reaction Time } \\
\% \text { Error }\end{array}$ & $\begin{array}{r}1,209 \\
503 \\
1.4\end{array}$ & $\begin{array}{r}1,268 \\
506 \\
2.4\end{array}$ & & \\
\hline \multicolumn{5}{|c|}{ Four-Alternative/Four-Cue } \\
\hline $\begin{array}{l}\text { Initiation Time } \\
\text { Reaction Time } \\
\% \text { Error }\end{array}$ & $\begin{array}{r}1,489 \\
509 \\
1.4\end{array}$ & & & $\begin{array}{r}1,432 \\
520 \\
2.4\end{array}$ \\
\hline \multicolumn{5}{|c|}{ Four-Alternative/Eight-Cue } \\
\hline $\begin{array}{l}\text { Initiation Time } \\
\text { Reaction Time } \\
\text { \% Error }\end{array}$ & $\begin{array}{r}1.496 \\
500 \\
2.8\end{array}$ & $\begin{array}{r}1,572 \\
543 \\
4.2\end{array}$ & $\begin{array}{r}1,704 \\
569 \\
1.4\end{array}$ & $\begin{array}{r}1,585 \\
519 \\
6.9\end{array}$ \\
\hline
\end{tabular}


tainty, the effect of stimulus uncertainty is substantially smaller than the initiation effects obtained here (Biederman \& Zachary, 1970). Thus, it is unlikely that the initiation-time result was due entirely to cue uncertainty.

However, this experiment rules out only one class of accounts of the initiation-time effect. Another alternative might be that two kinds of interpretive processing occur after the initial coding: first, the relevant information is extracted from the cue representation and translated into some more accessible form, and then the transformed cue representation is used to select strategy features and prepare the strategy representation. In this view, some or all of the observed effects may have been due to the translation process rather than to strategy preparation. (Reeve \& Proctor, 1984, describe a similar criticism of some movement precuing results.) The present results suggest that the initiation-time effects are due to some kind of interpretive processing, rather than initial encoding operations, but do not indicate the nature of that interpretive processing. Thus, we can say only that the effect arises somewhere in the process of using the information in the cue to specify a strategy.

\section{EXPERIMENTS 4 AND 5}

Experiments 1-3 demonstrated that the strategy cuing technique can be used to study strategy preparation. They showed that an increase in trial-initiation time occurs when more features have to be specified, that the increase is not due to uncertainty about which strategy to use, and that the increase is not due to cue encoding operations. Experiments 4 and 5 investigated whether the initiationtime results could be explained by assuming that the features of a strategy are specified in series, and that the time to prepare the entire strategy is related to the sum of the feature-specification times.

In this model, we make a number of simplifying assumptions about the process of specifying a strategy. To begin with, we assume that features are specified independently. Second, we assume that specifying each feature constitutes a discrete processing stage in the sense of Sternberg (1969). Third, we assume that the process of specifying a feature does not vary much in duration when compared with the initiation-time effects (see Townsend, 1984). Finally, we assume that the strategy features can be specified in any order (cf. Rosenbaum, 1983). Together, these assumptions predict that the factorial manipulation of features to be specified should produce additive effects. If the results are not additive, it would be appropriate to relax some of these assumptions, or to consider alternative models.

In Experiments 4 and 5, the task was changed so that there were three possible features that might have to be specified on a given trial. The new task was similar to that of Rosenbaum (1980) and involved making response movements either forward or backward to either a near target or a far target. Thus, in addition to the laterality and extent features used in Experiment 1, there was also a movement direction feature that could be specified in the strategy. In Experiment 4, direction and laterality were combined factorially across conditions; the specification of extent was always required. In Experiment 5, extent and laterality were combined factorially, and direction was always required. The serial model predicts that, in both experiments, feature-specification times should be additive.

\section{Method}

Apparatus and Procedure. The apparatus and procedure were generally the same as in Experiment 1; subjects sat in front of an inclined panel with two columns of switches. Subjects rested their index fingers on home switches in the middle of each column instead of at the bottom. There were two switches above and two below each home switch. Cues and stimuli were shown on a 30$\mathrm{cm}$ video monitor.

Stimuli. The cues were similar to those in Experiment 1 in that " $\mathrm{X}$ " and " $\mathrm{O}$ " indicated the $\mathrm{X}$ and $\mathrm{O}$ switches, and dashes indicated unused switches. Small filled rectangles stood for the positions of the home switches in the middle of each column. In other respects, the cue and stimulus displays were the same as in Experiment 1.

Conditions. In both experiments, strategies varied in terms of whether the " $X$ " and " $O$ " responses used the near switches next to the home switch or the further response switches (the extent feature), whether the " $\mathrm{X}$ " and " $\mathrm{O}$ " responses were movements forward or backward from the home switches (the direction feature), and whether the " $X$ " response was on the left or right side (the laterality feature). (As before, the stimulus-response mappings were always symmetric.) In Experiment 4, four conditions were constructed that varied in whether or not there was uncertainty about the laterality and direction features before seeing the cue on each trial. The four conditions were extent, direction/extent, laterality/extent, and direction/laterality/extent. (There was uncertainty about the extent feature in all four conditions.) Experiment 5 tested four analogous conditions: direction, direction/extent, direction/laterality, and direction/laterality/extent. The direction/extent and the direction/laterality/extent conditions were replications of conditions in Experiment 4.

In both experiments, conditions consisted of two blocks of 32 trials in which each cue and stimulus occurred equally often in a random order. In conditions with fixed laterality, one of the two blocks (chosen at random) had the $\mathrm{X}$ on the left and the $\mathrm{O}$ on the right, whereas the other conditions had the reverse mapping. Similarly, in conditions with fixed direction, one of the two blocks used forward responses and the other used backward responses. Trials and cues were classified in terms of the type of response with which they were associated (i.e., near forward, far forward, near backward, and far backward). Each condition began with 15 randomly selected practice trials.

Subjects. Subjects were undergraduates at the University of Alberta, 25 in Experiment 4 and 16 in Experiment 5. Data from 1 subject in Experiment 4 were not used because of a large number of very long initiation times, with the mean in some conditions being greater than five sec. Subjects in both experiments were divided into four groups, each with a different order of the four conditions. Across groups, each condition occurred in each serial position an equal number of times.

Analyses. Analyses of the initiation, reaction, and movement times were carried out on the means for correct trials for each subject, condition, and response type. In Experiment 5, two of the conditions used only near responses, so the principal analyses com- 
pared conditions only among these responses. Outliers greater than three standard deviations above the means were excluded. In Experiment 4 , these constituted $1.3 \%$ of the initiation times, $0.9 \%$ of the response times, and $0.6 \%$ of the movement times. In Experiment 5 , these constituted $1.7 \%$ of the initiation times, $0.6 \%$ of the reaction times, and $1.1 \%$ of the movement times.

\section{Results}

Initiation times. The results are shown in Figures 6 and 7 . In both experiments, there was a clear failure of additivity for initiation times. In Experiment 4, there was an effect of laterality $[F(1,20)=8.76, p<.01]$ and an interaction of laterality and direction $[F(1,20)=5.50$, $p<.05]$. The interaction was caused by long initiation in the direction/extent condition relative to the extent condition $[F(1,20)=3.90, p=.06]$, but similar initiation times in the direction/laterality/extent condition and the laterality/extent condition $[F(1,20)=1.03]$. The fact that the direction/laterality/extent condition actually required

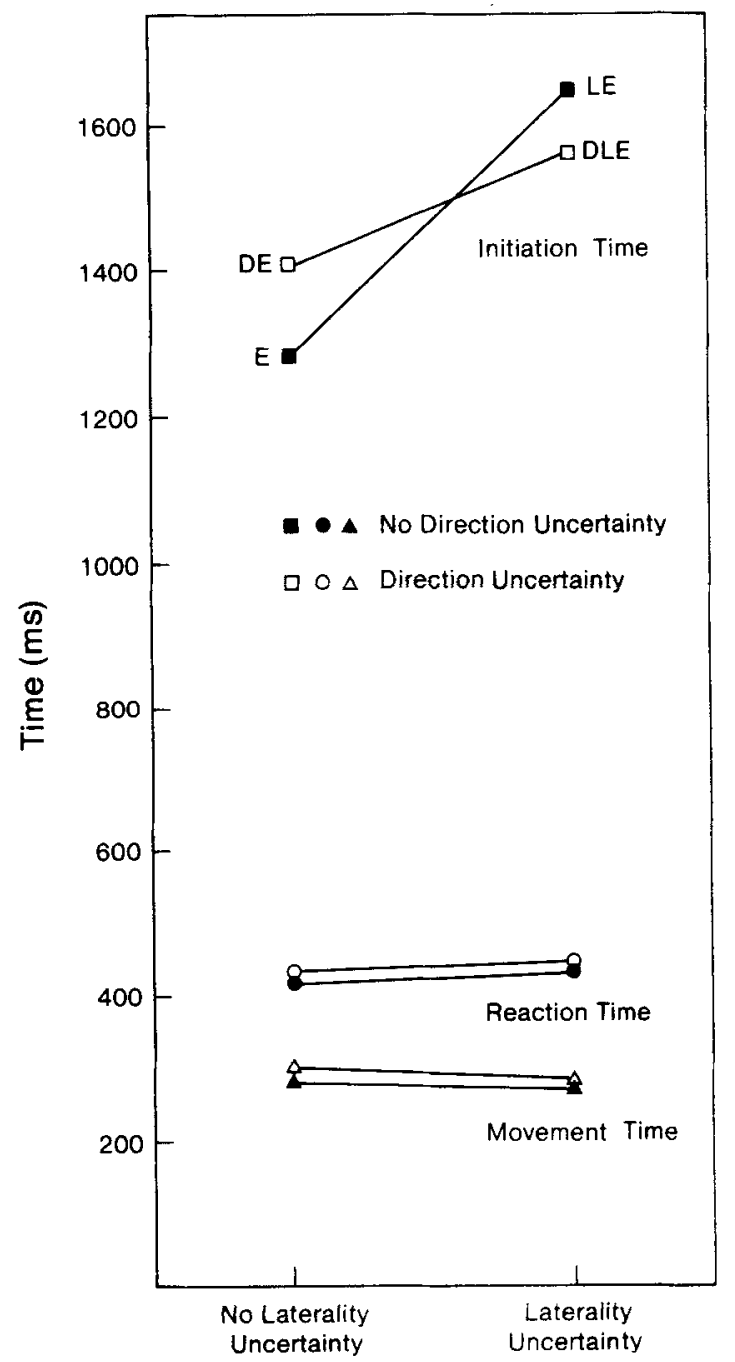

Figure 6. Mean initiation, reaction, and movement times in Experiment $4(E=$ extent condition; DE $=$ direction/extent condition; $\mathbf{L E}=$ laterality/extent condition; DLE $=$ direction/laterality/extent condition.

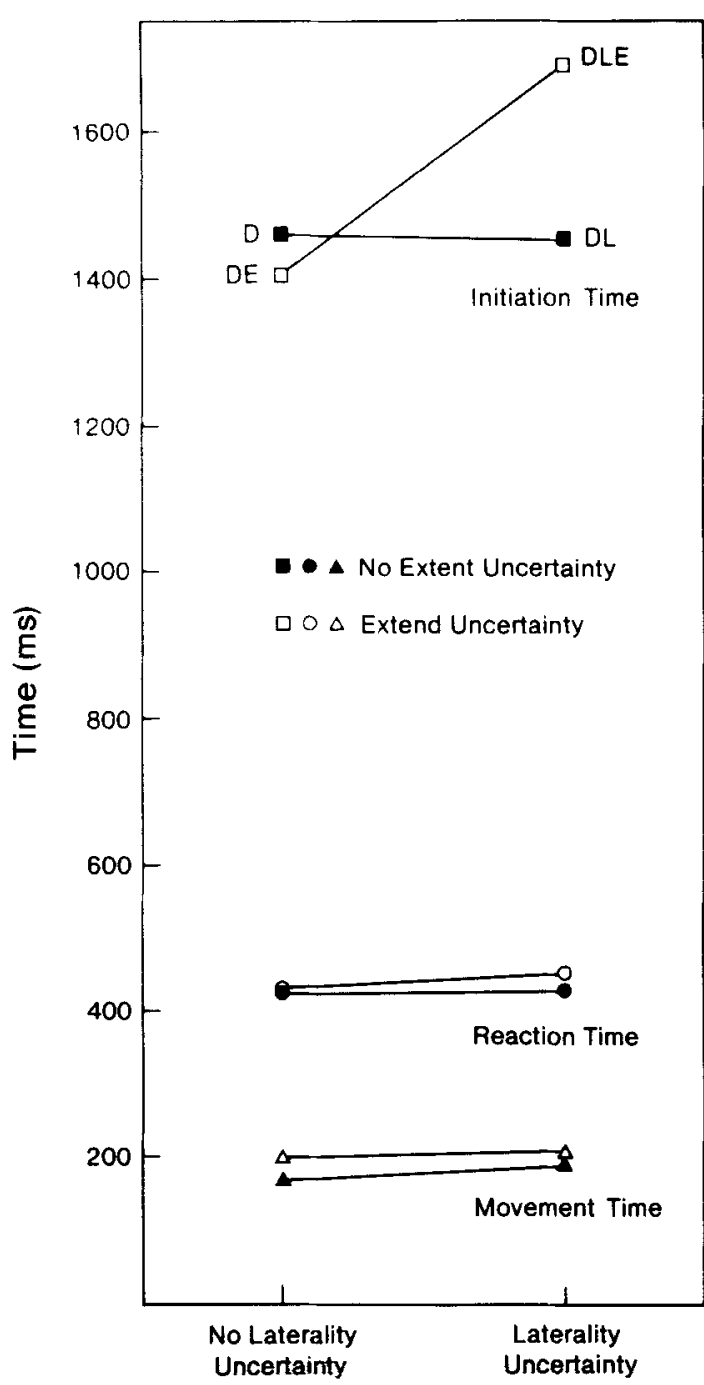

Figure 7. Mean initiation, reaction, and movement times for near response trials in Experiment $5(D=$ direction condition; $D E=$ direction/extent condition; DL = direction/laterality condition; DLE = direction/laterality/extent condition).

less time than the laterality/extent condition should probably be attributed to sampling error; there was no significant difference between the two conditions in a similar experiment reported by Dixon (1979), but the direction/laterality/extent condition yielded the longer initiation times. There was also an effect of trial type in Experiment $4[F(3,60)=3.88, p<.05$; see Table 5].

In Experiment 5, the only difference among initiation times was that the direction/extent/laterality condition had longer initiation times than the other three conditions. This led to an interaction between the laterality and extent factors $[F(1,12)=6.22, p<.05]$. This comparison involved near response trials only; a comparison of the far response trials showed a similar difference between the direction/laterality/extent condition and the direction/extent condition $[F(1,12)=8.45, p<.05]$.

Reaction and movement times. Reaction times showed no differences across conditions in Experiment 4, but in Experiment 5 showed effects of laterality $[F(1,12)=$ 
$7.30, p<.05]$ and direction $[F(1,12)=6.06, p<.05]$. These effects may have been due to initiation time "errors." That is, some subjects may have carried over some of their strategy preparation into the reaction and movement intervals because they terminated their initiation time too soon. This tendency was apparently stronger in Experiment 5. Movement times showed an effect of extent uncertainty in Experiment $5[F(1,12)=8.10, p<.05]$.

The effects of trial type are shown in Tables 5 and 6. In both experiments, there were significant differences for reaction and movement times $[F(3,60)=12.77, p<$ .001 , and $F(3,60)=31.14, p<.001$ for Experiment 4; $F(3,36)=5.92, p<.01$, and $F(3,36)=26.90, p<$ .001 for Experiment 5].

Errors. Error rates are also shown in Tables 5 and 6. There were no significant differences across conditions. However, in Experiment 4, errors were related to the position being moved to $[F(3,60)=7.98, p<.001]$.

\section{Discussion}

The important result of these experiments was the presence of interactions between laterality and direction uncertainty, and between laterality and extent uncertainty. Thus, the time to specify a strategy does not seem to be simply the sum of the time to specify each of a number of features, and a simple serial model of the featurespecification process appears not to be appropriate. We propose instead a mixed serial-parallel model of the specification process. In this mixed model, we assume that extent and laterality are specified serially, and that direction is specified in parallel with other features. We assume further that specifying direction takes more time than specifying either extent or laterality alone, but less time than specifying both laterality and extent in series. This mixed model departs minimally from the simple serial model, yet is sufficient to account for the results of Experiments 4 and 5.

In Experiment 4, the shortest initiation times occur in the extent condition because only a single feature is specified. Because the two features in the direction/extent condition are specified in parallel, the total initiation time is determined by the longer direction feature. Even longer times occur in the laterality/extent and direction/laterality/extent conditions because they both require extent and laterality features to be specified one after the other. The model predicts that the initiation time should be similar

Table 5

Times (in Milliseconds) and Error Rates by Trial Type in Experiment 4

\begin{tabular}{lcccc} 
& \multicolumn{4}{c}{ Trial Type } \\
\cline { 2 - 5 } Condition & $\begin{array}{c}\text { Near } \\
\text { Forward }\end{array}$ & $\begin{array}{c}\text { Far } \\
\text { Forward }\end{array}$ & $\begin{array}{c}\text { Near } \\
\text { Backward }\end{array}$ & $\begin{array}{c}\text { Far } \\
\text { Backward }\end{array}$ \\
\hline Initiation Time & 1,178 & 1,399 & 1,473 & 1,538 \\
Reaction Time & 434 & 439 & 416 & 406 \\
Movement Time & 251 & 306 & 242 & 309 \\
\% Error & 7.9 & 9.6 & 11.3 & 13.2 \\
\hline
\end{tabular}

Table 6

Times (in Milliseconds) and Error Rates in Experiment 5

\begin{tabular}{lrrrr}
\hline & \multicolumn{4}{c}{ Trial Type } \\
\cline { 2 - 5 } Condition & $\begin{array}{c}\text { Near } \\
\text { Forward }\end{array}$ & $\begin{array}{c}\text { Far } \\
\text { Forward }\end{array}$ & $\begin{array}{c}\text { Near } \\
\text { Backward }\end{array}$ & $\begin{array}{c}\text { Far } \\
\text { Backward }\end{array}$ \\
\hline Direction & & & & \\
Initiation Time & 1,477 & & 1,435 & \\
Reaction Time & 411 & & 411 & \\
Movement Time & 175 & & 160 & \\
\% Error & 7.8 & & 5.7 & \\
Direction/Extent & & & & \\
Initiation Time & 1,436 & 1,410 & 1,368 & 1,384 \\
Reaction Time & 427 & 413 & 417 & 399 \\
Movement Time & 190 & 241 & 192 & 265 \\
\% Error & 6.6 & 5.9 & 5.9 & 8.2 \\
Direction/Laterality & & & & \\
Initiation Time & 1,453 & & 1,441 & \\
Reaction Time & 427 & & 426 & \\
Movement Time & 189 & & 172 & \\
\% Error & 7.4 & & 5.7 & \\
Direction/Laterality/Extent & & & \\
Initiation Time & 1,710 & 1,652 & 1,658 & 1,685 \\
Reaction Time & 456 & 454 & 455 & 438 \\
Movement Time & 207 & 250 & 185 & 261 \\
\% Error & 5.1 & 3.9 & 6.3 & 8.2 \\
\hline
\end{tabular}

in these two conditions, consistent with the nonsignificant difference obtained in Experiment 4.

In Experiment 5, direction, direction/laterality, and direction/extent all had similar initiation times. Because direction is specified in parallel with extent and laterality, and is assumed to take longer than either one alone, the total initiation time would be determined by the time to specify direction alone. The long initiation times found in the direction/laterality/extent condition replicate the similar effect in Experiment 4 and are explained in the same way: Because extent and laterality are specified serially, together they take longer than the direction feature specified in parallel. In sum, the mixed serial-parallel model accounts for the results of Experiments 4 and 5 by assuming that initiation time in any condition requiring a direction feature will be longer than any single-feature condition, and that initiation time in conditions with both laterality and extent features will be longest of all.

\section{GENERAL DISCUSSION}

For the five experiments in this report, we used trialinitiation time to investigate the process of specifying strategies in a choice-reaction task. These experiments have provided several important results on how subjects specify strategies varying in stimulus-response mappings. One such result is that the number of alternative strategies has surprisingly little direct effect on strategypreparation time. One might have conjectured that uncertainty would affect strategy specification even when the number of strategy features was held constant. Such a result would be in line with research on choice reactions 
(e.g., Hick, 1952), absolute judgments (e.g., Garner, 1953), and motor responses (Fitts \& Peterson, 1964). Nevertheless, only one of the experiments provided any initiation-time effects that could not be ascribed to the number of strategy features. That was the result for Type 2 trials in Experiment 2; a difference was found between four alternatives and eight, even though the number of features was the same in both. However, as pointed out earlier, that difference may have been due to the relative discriminability of the cues rather than uncertainty per se. Overall, if uncertainty does have an effect on strategy preparation, it would seem to be too small to be detected with this paradigm.

In contrast to uncertainty, the number of strategy features had a systematic and robust effect on trial-initiation time. In Experiments 1,2, and 3, a substantial increment was found when an extent feature or a finger feature had to be specified. In Experiments 4 and 5, increments were also found for specifying laterality features and for direction features under some conditions. Thus, the number and nature of the features seems to be a principal determinant of the time needed to specify a strategy in this task.

A potential problem in interpreting initiation times is that they may include the time to make small preparatory movements. For instance, if a cue indicates that responses will require a forward movement, subjects may move their wrists forward slightly in anticipation of the response, even though their fingers are still in contact with the home switch. Subjects were asked not to make such movements, and none were observed in the experiments, but that is no guarantee that they did not occur. Moreover, such movements may have contributed differentially to initiation times in different conditions. A wrist adjustment might have been made on many trials in conditions where direction changes from trial to trial, but not in conditions where the direction of movement was always the same. Thus, the difference in initiation time between conditions with direction uncertainty and conditions without direction uncertainty may be partially due to these preparatory movements.

It should be pointed out that such movements are in themselves evidence that a cognitive strategy has been prepared. If a subject moves his or her hand in preparation for making a forward response, it means that he or she has already decided to make that kind of response. Essentially, a cognitive strategy feature must be specified prior to any corresponding motor adjustments. However, the preparatory movements may make it difficult to estimate how long the cognitive component of the strategy specification takes; it is always possible that an estimate is partly an estimate of how long it takes to make the preparatory movement.

Of the strategy features studied in these experiments, direction and finger features seem to be the most susceptible to motor involvement. As pointed out above, specifying direction might involve subjects' moving their wrists slightly forward or backward when anticipating a forward or backward response. In addition, the finger feature in Experiment 2 might involve subjects' tensing of the appropriate response fingers, or slight lifting of the other fingers. However, it is difficult to imagine that any motor preparation is associated with extent features or laterality features. The duration estimates for these features are likely to involve purely cognitive operations.

In conclusion, this research has demonstrated a number of important constraints on how people prepare strategies for speeded tasks. These findings are important because they concern the process of preparing the strategy, rather than simply the nature of the strategy itself. This issue requires much more investigation in cognitive psychology. Although the present research was limited to a relatively small number of strategy features, it indicates how temporal aspects of strategy preparation can be investigated systematically, and provides a framework for a much broader investigation.

\section{REFERENCES}

Biederman, I., \& Zachary, R. A. (1970). Stimulus versus response probability effects in choice reaction time. Perception \& Psychophysics, $7,189-192$.

Davis, R. (1964). The combination of information from different sources. Quarterly Joumal of Experimental Psychology, 16, 332-339.

DiXON, P. (1979). The selection and modification of algorithms in speeded tasks. Dissertation Abstracts International, 40, 5437B. (University Microfilms No. 8010927)

Dixon, P. (1981). Algorithms and selective attention. Memory \& Cognition, 9, 177-184.

Fitts, P. M., \& Peterson, J. R. (1964). Information capacity of discrete motor responses. Journal of Experimental Psychology, 67, 103-112.

GARNER, W. R. (1953). An information analysis of absolute judgments of loudness. Journal of Experimental Psychology, 46, 373-380.

Goodman, D., \& Kelso, J. A. S. (1980). Are movements prepared in parts? Not under compatible (naturalized) conditions. Journal of Experimental Psychology: General, 109, 475-495.

HICK, W. E. (1952). On the rate of gain of information. Quarterly Journal of Psychology, 4, 11-26.

KeEle, S. W., Summers, J. J. (1976). The structure of motor programs. In G. E. Stelmach (Ed.), Motor control: Issues and trends (pp. 109-142). New York: Academic Press.

Leonard, J. A. (1958). Partial advance information in a choice reaction task. British Journal of Psychology, 49, 89-96.

LoGAN, G. D., \& ZBrodofF, N. J. (1982). Constraints on strategy construction in a speeded discrimination task. Journal of Experimental Psychology: Human Perception \& Performance, 8, 502-520.

Logan, G. D., Zbrodoff, N. J., \& Fostey, A. R. W. (1983). Costs and benefits of strategy construction in a speeded discrimination task. Memory \& Cognition, 11, 485-493.

MEGAw, E. D. (1972). Direction and extent uncertainty in step-input tracking. Journal of Motor Behavior, 4, 171-186.

Merikle, P. M., \& Coltheart, M. (1972). Selective forward masking. Canadian Journal of Psychology, 26, 296-302

Reeve, T. G., \& Proctor, R. W. (1984). On the advance preparation of discrete finger responses. Journal of Experimental Psychology: $\mathrm{Hu}$ man Perception \& Performance, 10, 541-553.

Rosenbaum, D. A. (1980). Human movement initiation: Specification of arm, direction and extent. Journal of Experimental Psychology: General, 109, 444-474.

Rosenbaum, D. A. (1983). The movement precuing technique: Assumptions, applications, and extensions. In R. A. Magill (Ed.), Movement and control of action (pp. 231-274). Amsterdam: North-Holland. 
SHAFFER, L. H. (1966). Some effects of partial advance information on choice reaction with fixed or variable S-R mapping. Journal of Experimental Psychology, 72, 541-545.

Shiffrin, R. M., \& Schneider, W. (1977). Controlled and automatic human information processing: II. Perceptual learning, automatic attending, and a general theory. Psychological Review, 84, 127-190.

SMrTH, E. E. (1968). Choice reaction time: An analysis of the major theoretical positions. Psychological Bulletin, 69, 77-110.

STERNBERG, S. (1969). The discovery of processing stages: Extensions of Donders' method. Acta Psychologica, 30, 276-315.
Townsend, J. T. (1984). Uncovering mental processes with factorial experiments. Mathematical Psychology, 28, 363-400.

ZelaZNIK, H. (1978). Precueing response factors in choice reaction time: A word of caution. Journal of Motor Behavior, 10, 77-79.

Zelaznik, H. N., Shapiro, D. C., \& CarTer, M. C. (1982). The specification of digit and duration during motor programming: A new method of precueing. Journal of Motor Behavior, 14, 57-68.

(Manuscript received February 14, 1985; revision accepted for publication April 29, 1986.) 